\title{
Factors associated with successful publication of research abstracts presented at the Japanese Society of Anesthesiologists annual meetings 2015-2017: a bibliometric analysis
}

\author{
Yuko Ono ${ }^{1,2, *}$, Masafumi Saito ${ }^{1}$, Chiaki Shinohara ${ }^{3}$, Kazuaki Shinohara ${ }^{2}$, \\ Shigeaki Inoue ${ }^{1}$, Joji Kotani ${ }^{1}$
}

\author{
${ }^{1}$ Department of Disaster and \\ Emergency Medicine, Graduate School \\ of Medicine, Kobe University, 7-5-2 \\ Kusunoki-cho, Chuo-ward, Kobe, Hyogo \\ 650-0017, Japan \\ ${ }^{2}$ Department of Anesthesiology, Ohta \\ General Hospital Foundation, Ohta \\ Nishinouchi Hospital, 2-5-20 \\ Nishinouchi, Koriyama, Fukushima \\ 963-8558, Japan \\ ${ }^{3}$ Library for Medical Sciences, School of \\ Medicine, Kobe University, 7-5-2 \\ Kusunoki-cho, Chuo-ward, Kobe, Hyogo \\ 650-0017, Japan
}

\section{*Correspondence}

windmill@people.kobe-u.ac.jp

(Yuko Ono)

\begin{abstract}
Background: Publication in a scientific journal is the desired outcome of the research cycle. However, previous anesthesiology research has not thoroughly examined factors predictive of subsequent publication after a meeting presentation. We aimed to assess the rate of peer-reviewed publication of abstracts presented at the Japanese Society of Anesthesiologists (JSA) annual meetings and identify factors associated with successful publication.

Methods: This study included all abstracts presented at JSA meetings from 2015 to 2017. The outcome of interest was subsequent publication of abstracts in journals included in the MEDLINE database within 36 months after presentation. Differences between published and non-published abstracts were evaluated.

Results: Among the 2,418 eligible abstracts, 487 were published within 3 years (publication rate, 20.1\%). Multivariable logistic regression analysis showed that factors independently associated with subsequent publication were: presentation style (poster discussions: adjusted odds ratios (AOR) 1.70, 95\% confidence interval [CI] 1.31-2.20; excellent abstracts: AOR 2.82, 95\% CI 1.98-4.01); basic research (AOR 4.39, 95\% CI 3.23-5.96), male first author (AOR 1.41, 95\% CI 1.09-1.81); region (Kansai: AOR 2.16, 95\% CI 1.57-2.99; abroad: AOR 4.57, 95\% CI 2.58-8.09); facility characteristics (private university: AOR 3.97, 95\% CI 2.60-6.08; public university: AOR 3.53, 95\% CI 2.35-5.30; medical company: AOR 16.70, 95\% CI 3.75-74.46); and number of collaborating facilities (two: AOR 1.50, 95\% CI 1.15-1.97; three: AOR 1.83, 95\% CI 1.23-2.73; four: AOR 2.40, 95\% CI 1.27-4.54).

Conclusions: Approximately one-fifth of abstracts presented at JSA meetings are published in peer-reviewed journals within 3 years. Factors independently associated with subsequent full publication are presentation style, basic research, male gender, specific region, affiliation characteristics, and number of collaborating facilities. Our data should stimulate further studies that elucidate ways to assist the full publication process of meeting abstracts.
\end{abstract}

\section{Keywords}

Abstract publication rate; Academic conference; Bibliometric analysis; Gender gap; MEDLINE database

\section{Introduction}

Results of biomedical research are usually summarized in abstract form and presented at scientific meetings. Professional meetings offer a platform for exchanging scientific information from the latest research, building scientific networks, and developing new ideas for future studies. However, scientific meetings are insufficient to disseminate research findings. For example, although some scientific societies publish meeting abstracts on the Internet, these abstracts do not appear in
MEDLINE or other electronic databases. Furthermore, some journals limit the citation of abstracts as references to a specified time after publication of the abstract. Conference abstracts that never achieved full publication are referred to as lost information [1]. In general, scientific information contained in meeting abstracts is assumed to be subsequently published in a peer-reviewed journal in full manuscript form within a reasonable period. Therefore, the rate of publication of meeting abstracts is considered an indicator of the scientific 
quality of a meeting [2, 3]. A recent meta-analysis of full publication following abstract presentations that included 425 studies found publication rates ranged from $2.6 \%$ to $90.7 \%$ (median rate $37.3 \%$ ) [4].

In terms of anesthesiology as a specialty, abstract to publication rates for presentations at conferences held by associations such as the International Anesthesia Research Society, American Society of Anesthesiologists, and European Society of Anaesthesiologists have been reported to range from $0 \%$ to $53.6 \%$ [5-13]. Approximately 10,000 anesthesiologists from around the world attend the Japanese Society of Anesthesiologists (JSA) meeting each year and several hundred research abstracts are presented. This represents a unique opportunity to learn about novel research findings in anesthesiology. Although estimating the scientific level and output of such a large professional convention is important, there are no published data reporting the subsequent full publication rate of abstracts presented at JSA meetings. Serious concerns regarding the steep decline in anesthesia journal publications from Japan have recently been raised [14-18]. Therefore, we need to advance our understanding of current research output, especially from Japan. In addition, previous anesthesiology research [5-13] did not thoroughly examine factors predictive of subsequent publication after a meeting presentation. To promote the publication process of scientific meeting abstracts, factors associated with successful publication should be elucidated.

In this study, we sought to determine the fate of abstracts presented at JSA meetings from 2015 to 2017 by focusing on two objectives: assessing the publication rate in peer-reviewed journals and identifying factors associated with successful publication. We believe this study will provide information to facilitate the full publication process after scientific meeting presentations.

\section{Methods}

\subsection{Study design, setting, subjects, and data source}

This bibliometric analysis was conducted from January 2020 to August 2020 at a public university in Japan. All research abstracts accepted for presentation at JSA meetings from 2015 to 2017 were extracted from the official JSA members' website in May 2020. Abstracts from symposia, problem-based learning discussions, refresher course seminars, sponsored seminars, and other invited lectures were excluded from the analysis. Journal articles that were retracted after publication were also excluded.

\subsection{Ethics approval and consent to participate}

Current Japanese ethical guidelines do not mandate ethical review for studies analyzing publicly available data. This study was therefore exempt from ethical evaluation by the Institutional Review Board at the authors' facilities. Similarly, as this was a bibliometric analysis that did not involve human subjects, the need for informed consent was waived.

\subsection{Outcome measures}

The main outcome of interest was the subsequent publication of JSA meeting abstracts in the journals included in the MEDLINE database within 36 months after presentation. PubMed ${ }^{\circledR}$ was used to search the MEDLINE database. Other outcomes of interest were factors predictive of publication and the time-course of peer-reviewed publications after presentation. The journals in which JSA meeting abstracts were ultimately published and the impact factors of those journals were also recorded. Cumulative full publication was assessed using the Kaplan-Meier method. We considered 36 months as a reasonable time from presentation to publication because most articles derived from presentations at scientific meetings are known to be published within 36 months [4]. Time to publication was rounded to the nearest month, and articles published in peer-reviewed journals before the JSA annual meeting where they were presented were given a zero value for time of publication. Journal impact factors were obtained using InCites Journal Citation Reports ${ }^{\mathrm{TM}}$ (Clarivate Analytics, Philadelphia, PA, USA).

\subsection{Assessment of subsequent publication}

By referencing relevant studies [3-13, 19-24], the search strategy was determined a priori by the survey team, which comprised anesthesiologists, a librarian, a basic scientist, and emergency physicians. To assess whether the abstract had been published, the method previously described by Tyagi et al. [9] and Macmillan et al. [21] was used with minor modifications. In brief, the names of authors' facilities and first and last authors were used as search keywords. First authors were used as they were most likely to have written the final paper, and last authors were used as they were also most likely to be last author in the final paper. If no corresponding paper was found, second authors were used instead of first authors. Japanese authors' names are usually written in kanji characters and may sometimes be difficult to read. To address this problem, we referred to the 2020 lists of JSA board-certificated anesthesiologists available on the JSA official members' website (https://anesth.or.jp/ users/member/certificate_information/doctors, accessed August 8, 2020). The 2020 lists cover 20,525 anesthesiologists and show how to read a member's name using katakana characters.

To evaluate the consistency between meeting abstracts and final publications, the methods previously reported by DeMola et al. [23] were employed with minor modifications. Briefly, we compared the similarity of the title, research hypothesis, study design, protocol, results, and conclusions between the original abstract and the final publication. Articles that contained all data presented in the abstract but also included additional data were regarded as a match, but publications that provided dissimilar data despite similar techniques or study designs were excluded. 
With the aid of a professional librarian (author CS), all eligible JSA meeting abstracts were initially evaluated by the first author (YO) in June 2020. The second author (MS) then replicated the search using the same strategy to check for errors and increase the reliability of the search. All discrepancies were resolved by mutual agreement.

\subsection{Statistical analysis}

To determine factors associated with subsequent full publication of JSA meeting abstracts, differences in characteristics between published and non-published abstracts were evaluated. We examined associations between various factors and full publication of abstracts, including: JSA annual meeting year $(2015,2016$, and 2017); presentation style (poster presentations, poster discussions, and excellent abstracts); presentation topics (cardiovascular medicine, respiratory medicine, neuroscience, pediatric anesthesia, obstetric anesthesia, pain medicine, intensive care unit [ICU] and emergency medicine, general topics in anesthesia, and anesthesia-related topics); research type (clinical or basic research); number of author(s) and authors' affiliation(s); gender of first author; first author's affiliation type (private community hospital, public community hospital, private university, public university, and medical company), and region of first author's affiliation (Kanto and Koshinetsu, Hokkaido and Tohoku, Tokai and Hokuriku, Kansai, Chugoku and Shikoku, Kyushu and Okinawa, and abroad).

We used the method previously reported by Demailly et al. [24] to identify the first author's gender. In short, the first author's gender was determined based on their first name. If the gender of the name was unclear, attempts were made to clarify the gender using search engines (e.g., Google Image ${ }^{\circledR}$ ) and institutional website profiles. Presentation topics were defined based on the "sessions" scheduled in the JSA meeting program. JSA annual meetings include three presentation styles. Usually, JSA reviewers assign the top $10 \%$ of abstracts to excellent abstracts, the following $30 \%$ of abstracts to poster discussions, and remaining abstracts to poster presentations.

Differences in categorical variables were assessed by chisquare tests followed by residual analysis. Univariable and multivariable logistic regression models were then fitted to yield crude and adjusted odds ratios (AOR) for successful publication. Inconsistent characteristics between the published and non-published abstracts (variables with $P<0.05$ in Table 1 , see the Results section) were included as independent variables in the logistic regression models.

In all multivariable analyses, a variance inflation factor was used to detect multicollinearity. We confirmed the goodness of fit and discrimination ability of the models with the Hosmer-Lemeshow test and the c-statistic, respectively. All statistical analyses were performed with SPSS Statistics for Windows, version 25.0 (IBM Corp., Armonk, NY, USA). The column scatter plot and Kaplan-Meier curve were generated using GraphPad Prism 8 (GraphPad Software, San Diego, CA, USA). A $P$-value $<0.05$ was considered to indicate statistical significance.

\subsection{Subgroup analysis}

We were particularly interested in how different presentation styles affected our outcomes of interest. Therefore, differences in the cumulative publication rate and journal impact factors of peer-reviewed publication of JSA meeting abstracts were analyzed separately according to presentation style. In addition to the statistical analysis described above, event rates of publication among the three presentation styles were compared using a log-rank test for the time to the first event after presentations. The difference in journal impact factors among these three groups was analyzed using a Kruskal-Wallis test followed by Dunn's post hoc tests with Bonferroni correction.

\section{Results}

\subsection{Publication rate}

Fig. 1 shows the study flow. Of the 3,256 JSA annual meeting abstracts from 2015 to 2017, we excluded 836 abstracts that were presented at symposia, problem-based learning discussions, refresher course seminars, sponsored seminars, and other invited lectures. The remaining 2,420 abstracts underwent PubMed $\AA$ searching. We found that 489 meeting abstracts were ultimately published. Of those, we excluded two abstracts from the analysis that were retracted after publication. Therefore, the publication rate of JSA meeting abstracts was $20.1 \%(487$ of 2,418$)$.

\subsection{Time to publication}

Fig. 2 shows the estimated cumulative publication rate for JSA meeting abstracts. The median time to full publication after JSA meeting presentation was 13 months (interquartile range, 3-22 months). We also found that 81 (3.3\%) meeting abstracts were published in peer-reviewed journals before the JSA annual meeting in which they were presented.

\subsection{Journal impact factor}

Fig. 3 shows the distribution of the impact factors for journals in which JSA meeting abstracts were published. The median journal impact factor was 2.07 (inter quartile range 1.45-3.34), and the range was $0-15.9$. The numbers of papers published in journals with an impact factor $<1$, between 1 and 3 , and $>3$ were 67 (13.8\%), $266(54.6 \%)$, and 154 (31.6\%), respectively.

\subsection{Differences in characteristics between published and non-published abstracts}

Table 1 compares the demographic characteristics between published and non-published research abstracts presented at JSA annual meetings from 2015 to 2017 . Research abstracts that were presented as poster discussions and excellent abstracts were more likely to be published than poster presentations. Abstracts on neuroscience and ICU and emergency medicine had higher publication rates than those on other topics. We also found that basic research had a higher possibility of publication than clinical research. Analysis of authors' characteristics showed the number of authors (four) and male gender were associated with successful publication. Similarly, in terms of affiliation characteristics, the number (two, three, 
TA B L E 1. Differences in characteristics between published and non-published research abstracts presented at the Japanese Society of Anesthesiologists annual meetings 2015-2017.

\begin{tabular}{|c|c|c|c|c|}
\hline & \multicolumn{4}{|c|}{ Research abstracts presented at JSA annual meetings 2015-2017 } \\
\hline & All Abstracts & Published abstracts ${ }^{a}$ & Non-published abstracts & $\boldsymbol{P}$ \\
\hline & $(\mathrm{N}=\mathbf{2 , 4 1 8})$ & $(\mathrm{N}=487)$ & $(\mathrm{N}=1,931)$ & \\
\hline JSA annual meeting year & & & & 0.984 \\
\hline 2015 & 854 & $173(20.3)$ & $681(79.7)$ & \\
\hline 2016 & 846 & $171(20.2)$ & $675(79.8)$ & \\
\hline 2017 & 718 & $143(19.9)$ & $575(80.1)$ & \\
\hline Presentation style & & & & $<0.001$ \\
\hline Poster presentations & 1,402 & $193(13.8) *$ & $1,209(86.2) * *$ & \\
\hline Poster discussions & 759 & $173(22.8) * *$ & $586(77.2) *$ & \\
\hline Excellent abstracts & 257 & $121(47.1) * *$ & $136(52.9) *$ & \\
\hline Presentation topics & & & & $<0.001$ \\
\hline Cardiovascular medicine & 299 & $65(21.7)$ & $234(78.3)$ & \\
\hline Respiratory medicine & 183 & $42(23.0)$ & $141(77.0)$ & \\
\hline Neuroscience & 95 & $49(51.6) * *$ & $46(48.4) *$ & \\
\hline Pediatric anesthesia & 91 & $11(12.1) *$ & $80(87.9) * *$ & \\
\hline Obstetric anesthesia & 144 & $20(13.9)$ & $124(86.1)$ & \\
\hline Pain medicine & 431 & $81(18.8)$ & $350(81.2)$ & \\
\hline ICU and emergency medicine & 156 & $48(30.8) * *$ & $108(69.2) *$ & \\
\hline Anesthesia-related topics & 766 & $146(19.1)$ & $620(80.9)$ & \\
\hline General topics in anesthesia & 253 & $25(9.9) *$ & $228(90.1) * *$ & \\
\hline Research type & & & & $<0.001$ \\
\hline Clinical research & 2,089 & $301(14.4) *$ & $1,788(85.6) * *$ & \\
\hline Basic research & 329 & $186(56.5) * *$ & $143(43.5) *$ & \\
\hline Number of the author(s) & & & & $<0.001$ \\
\hline One & 70 & $7(10.0) *$ & $63(90.0) * *$ & \\
\hline Two & 134 & $20(14.9)$ & $114(85.1)$ & \\
\hline Three & 259 & $51(19.7)$ & $208(80.3)$ & \\
\hline Four & 305 & $87(28.5) * *$ & $218(71.5) *$ & \\
\hline Five & 383 & $91(23.8)$ & $292(76.2)$ & \\
\hline Six & 1,267 & $231(18.2) *$ & $1,036(81.8) * *$ & \\
\hline Gender of the first author & & & & 0.006 \\
\hline Male & 1,545 & $337(21.8) * *$ & $1,208(78.2) *$ & \\
\hline Female & 873 & $150(17.2) *$ & $723(82.8) * *$ & \\
\hline Number of the author affiliation(s) & & & & $<0.001$ \\
\hline One & 1,519 & $234(15.4) *$ & $1,285(84.6) * *$ & \\
\hline Two & 614 & $164(26.7) * *$ & $450(73.3) *$ & \\
\hline Three & 208 & $62(29.8) * *$ & $146(70.2) *$ & \\
\hline Four & 58 & $20(34.5) * *$ & $38(65.5) *$ & \\
\hline Five or more & 19 & $7(36.8)$ & $12(63.2)$ & \\
\hline First author's affiliation type & & & & $<0.001$ \\
\hline Private community hospital & 548 & $38(6.9) *$ & $510(93.1) * *$ & \\
\hline Public community hospital & 499 & $42(8.4) *$ & $457(91.6) * *$ & \\
\hline Private university & 590 & $162(27.5) * *$ & $428(72.5) *$ & \\
\hline Public university & 768 & $235(30.6) * *$ & $533(69.4) *$ & \\
\hline Medical company & 13 & $10(76.9) * *$ & $3(23.1) *$ & \\
\hline First author's affiliation region & & & & $<0.001$ \\
\hline Kanto and Koshinetsu & 884 & $164(18.6)$ & $720(81.4)$ & \\
\hline Hokkaido and Tohoku & 181 & $35(19.3)$ & $146(80.7)$ & \\
\hline Tokai and Hokuriku & 283 & $45(15.9)$ & $238(84.1)$ & \\
\hline Kansai & 484 & $120(24.8) * *$ & $364(75.2) *$ & \\
\hline Chugoku and Shikoku & 245 & $50(20.4)$ & $195(79.6)$ & \\
\hline Kyushu and Okinawa & 264 & $30(11.4) *$ & $234(88.6) * *$ & \\
\hline Abroad & 77 & $43(55.8) * *$ & $34(44.2) *$ & \\
\hline
\end{tabular}

Data expressed as $n(\%) .{ }^{a}$ Defined as JSA meeting abstracts that were successfully published in the international journals

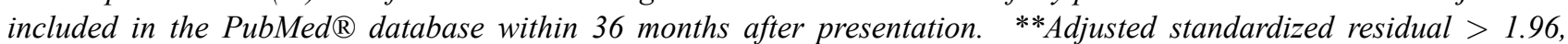
*adjusted standardized residual $<-1.96$. JSA, Japanese Society of Anesthesiologists. 


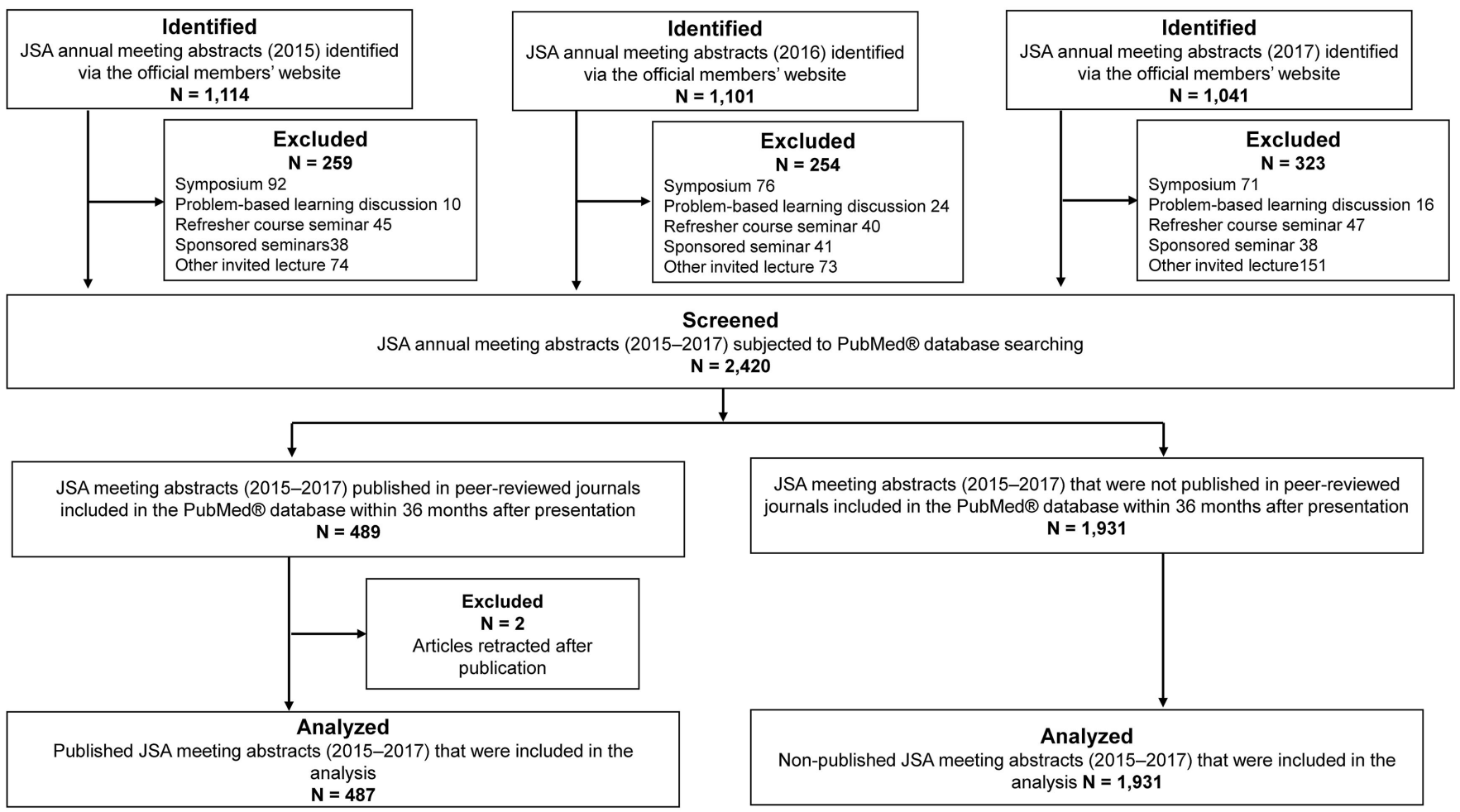

F I G U R E 1. Study flow diagram. JSA, Japanese Society of Anesthesiologists.

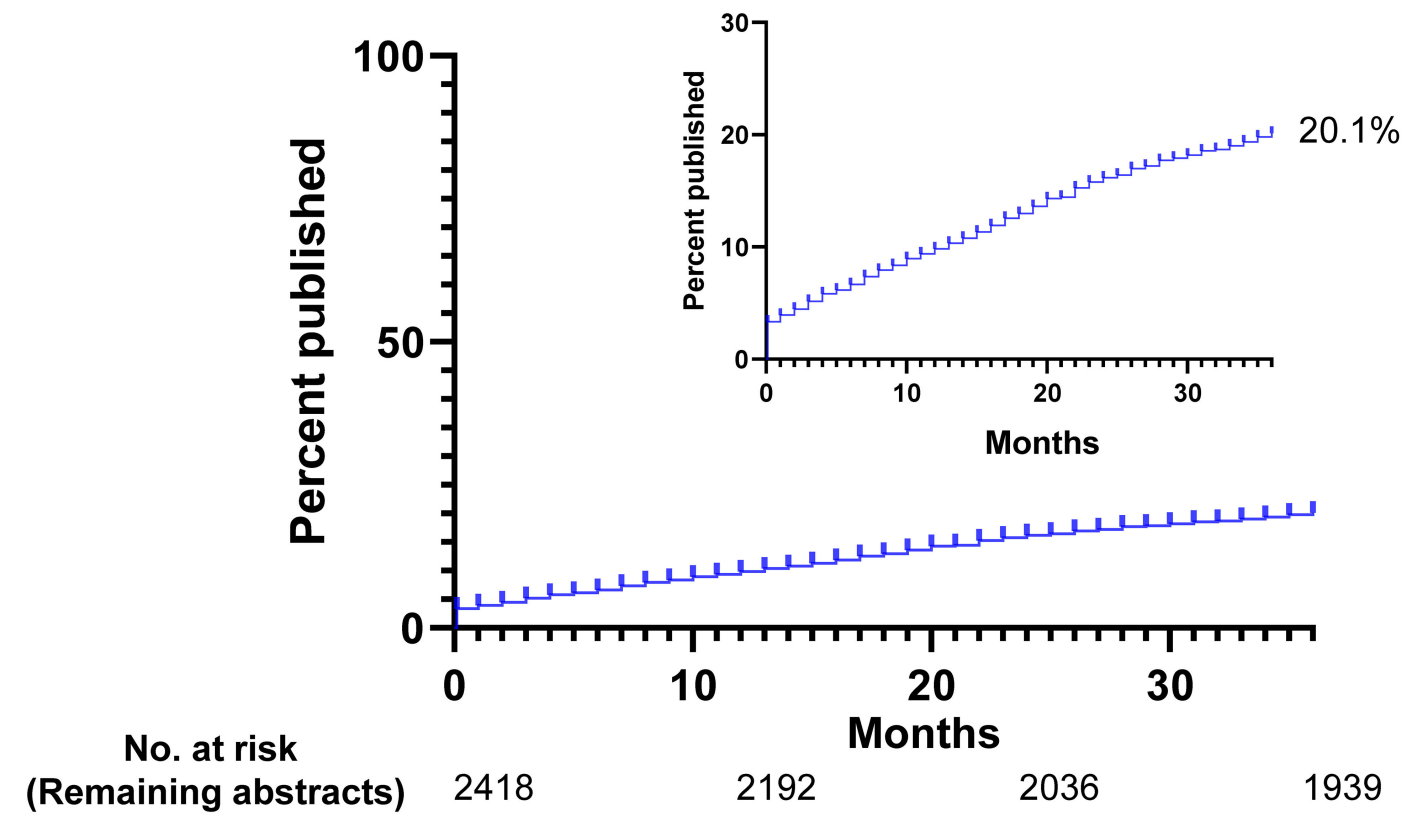

FIGURE 2. Cumulative full publication of research abstracts initially presented at the Japanese Society of Anesthesiologists annual meetings 2015-2017. Event rates shown are Kaplan-Meier estimates. The inset shows the same data on enlarged y axes. Among 2,418 research abstracts initially presented at JSA annual meetings 2015-2017, 487 (20.1\%) abstracts resulted in full publication within 36 months. Median time to full publication after JSA meeting presentation was 13 months (interquartile rage, 3-22 months), and 3.3\% (81 of 2,418) abstracts were published before the relevant JSA annual meeting. JSA, Japanese Society of Anesthesiologists.

and four), type (private university, public university, and medical company), and region (Kansai and abroad) of facilities were positively associated with publication.

\subsection{Factors associated with publication}

The results of the univariable and multivariable analyses for subsequent publication of JSA meeting abstracts are shown in Table 2. After adjustment in the multivariable logistic 


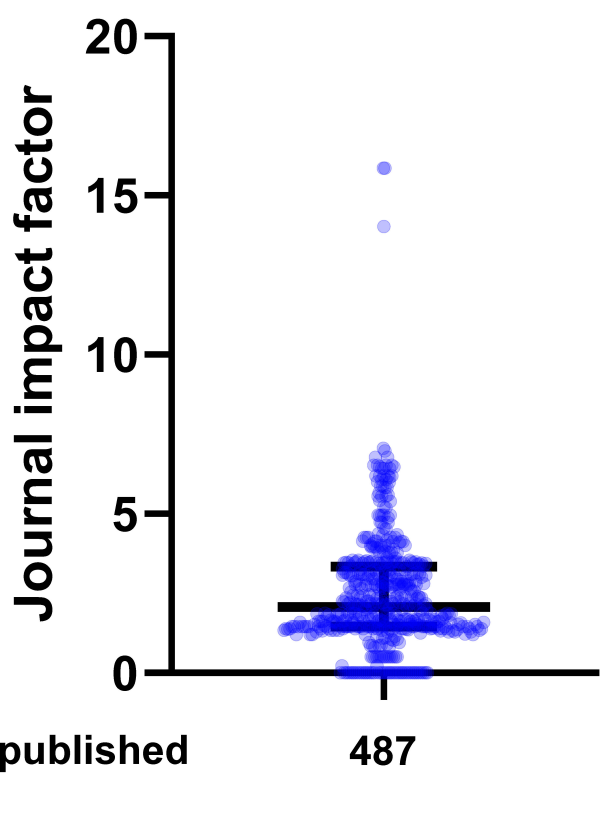

FIGURE 3. Journal impact factor of published research abstracts presented at the Japanese Society of Anesthesiologists annual meetings 2015-2017. Column scatter plots represent the data distribution (circles), median (horizontal bar), and interquartile range (vertical bar). The median journal impact factor was 2.07 (inter quartile range 1.45-3.34) and ranged from 0 to 15.9. The number of papers published in journals with an impact factor $<1$, between 1 and 3 , and $>3$, were 67 (13.8\%), $266(54.6 \%)$, and 154 (31.6\%), respectively.

regression model, factors independently associated with publication were: presentation style (poster discussions: AOR 1.70, 95\% confidence interval [CI] 1.31-2.20; excellent abstracts: AOR 2.82, 95\% CI 1.98-4.01), basic research (AOR 4.39, 95\% CI 3.23-5.96), male gender (AOR 1.41, 95\% CI 1.091.81), specific region (Kansai: AOR 2.16, 95\% CI 1.57-2.99; abroad: AOR 4.57, 95\% CI 2.58-8.09), affiliation characteristics (private university: AOR 3.97, 95\% CI 2.60-6.08, public university: AOR 3.53, 95\% CI 2.35-5.30; medical company: AOR 16.70, 95\% CI 3.75-74.46), and number of collaborating facilities (two: AOR 1.50, 95\% CI 1.15-1.97; three: AOR 1.83 , 95\% CI 1.23-2.73; four: AOR 2.40, 95\% CI 1.274.54). Multicollinearity was not detected (variance inflation factor of $<10.0$ for each explanatory variable). The HosmerLemeshow test verified the good fit of this model $(P=0.254)$, and the c-statistic for this model was 0.826 (95\% CI 0.806 $0.846)$, suggesting acceptable discrimination.

\subsection{Subgroup analysis}

As shown in Fig. 4, the cumulative publication rate significantly differed by presentation style (poster presentation, $13.8 \%$; poster discussions, 22.8\%; excellent abstracts, $47.1 \% ; P<0.001)$. Similarly, the journal impact factor for final publication was significantly higher for excellent abstracts than other two presentation styles (vs. poster presentation, $P<0.001$; and vs. poster discussions, $P$
$<0.01$, Fig. 5)

\section{Discussion}

Our analysis of more than 2,000 abstracts presented at JSA meetings (2015-2017) showed that approximately one-fifth of these abstracts were ultimately published in peer-reviewed journals within 3 years after presentation. The publication rate differed significantly according to presentation style, research type, authors' gender, affiliation characteristics, and number of collaborating facilities.

In this study, the subsequent publication rate of JSA meeting abstracts was $20.1 \%$. This abstract-publication rate was lower than that reported in other anesthesiology studies [5-13]. The reasons for those discrepancies may be multifactorial. Japan has fewer physicians per capita than most other OECD countries (https://www.oecdilibrary.org/sites/b33ab4c1-en/index.html? itemId=/content/component/b33ab4c1-en, accessed August 8, 2020). This means that many healthcare professionals (including anesthesiologists) in Japan are chronically exposed to a heavy workload. Most Japanese anesthesiologists also expected hold multifaceted roles encompassing clinical services, teaching residents, and ICU and operating room administration. Therefore, our study population may have difficulty finding time for research activity. This assumption was supported by the results that showed first the author's origin (abroad) and affiliation type (medical company) were both strong predictors of subsequent publication. In addition, the current JSA board certification system warrants mention. Anesthesiology residents entering specialty training programs need to make at least one presentation at a JSA annual meeting to apply for the board certification examination. Delivering a presentation at JSA annual meeting is also important for JSA board certified anesthesiologists to obtain points for retaining their license. Some JSA meeting presentations included in this study therefore might have been intended to obtain/maintain board certification, rather than subsequent publication in international journals. These differences in meeting focus along with differences in abstract selection processes, research culture, available funds/resources, data measurement, or a combination of these factors might have resulted in a relatively lower rate of anesthesiology publications in our study compared with previous studies. However, the fact that nearly $80 \%$ of JSA meeting abstracts did not achieve full publication warrants improvement.

This study also found that the first author being male was independently associated with full publication of the abstract. Japan lags behind other countries in gender equality. According to Global Gender Gap Report 2020 by World Economy Forum (http: //www3. weforum.org/docs/WEF_GGGR_2020. pdf, accessed August 8, 2020), Japan ranks 121st out of 153 countries in gender equality. In Japan, women physicians still spend more time than their male counterparts engaged in domestic work (e.g., child rearing and housekeeping) [25, 26]. Because the publication cycle requires a lot of time, women researchers in the field under study may have difficulty balancing research activity and family responsibilities. Our 


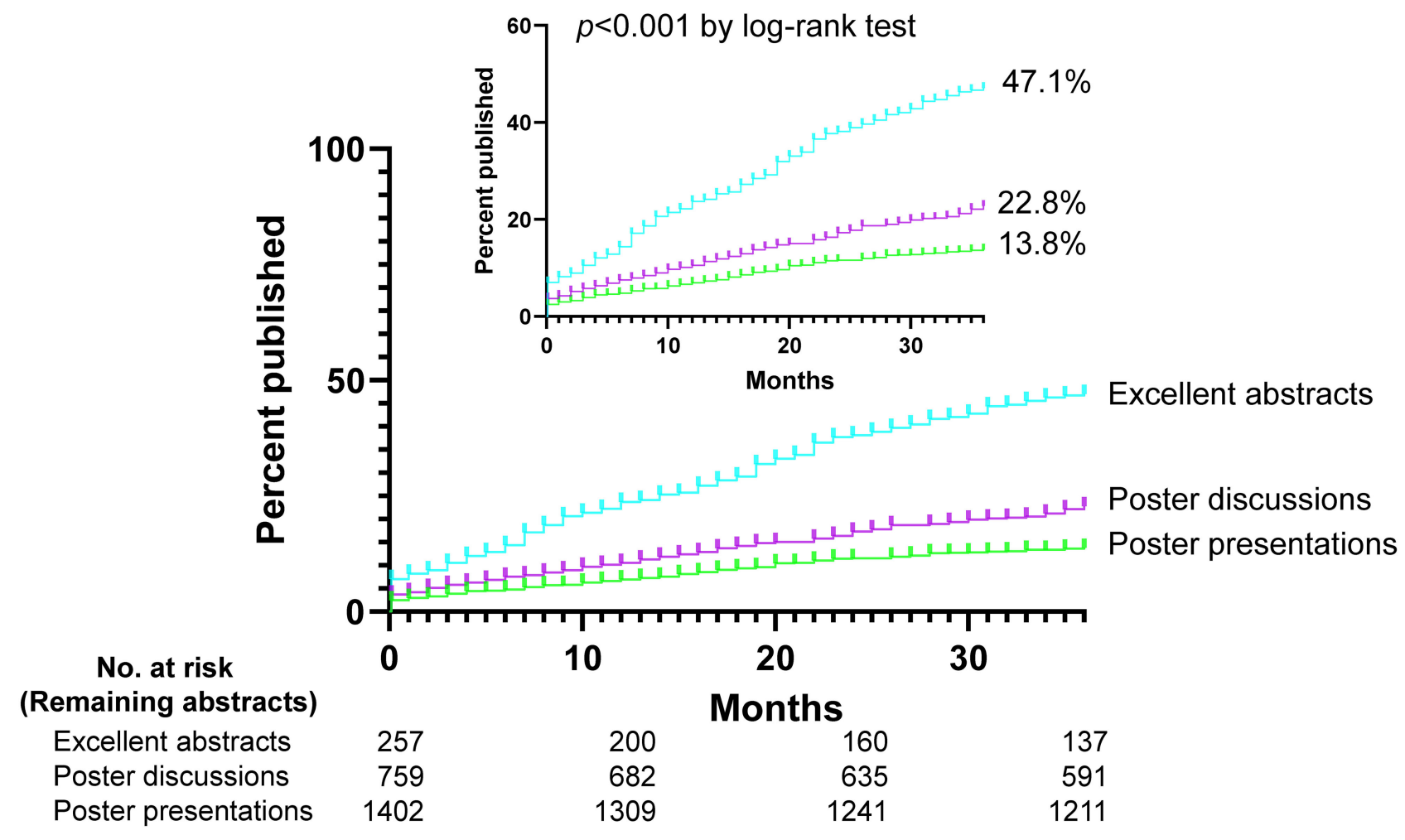

F I G U R E 4. Cumulative full publication of research abstracts by presentation style. Event rates shown are Kaplan-Meier estimates. The $P$-value was calculated using means of the log-rank test based on all available follow-up data. The inset shows the same data on enlarged y axes. The number of research abstracts presented as poster presentations, poster discussions, and excellent abstracts at the Japanese Society of Anesthesiologists annual meetings 2015-2017 were 1402, 759, and 257, respectively. Of those abstracts, 193 (13.8\%) poster presentations, 173 (22.8\%) poster discussions, and 121 (47.1\%) excellent abstracts resulted in full publication within 36 months.

finding was consistent with previous studies that reported a gender gap in academic achievement [27-30]. Increased institutional support and continuous, focused organizational efforts are needed to promote gender equality [27,30].

This study also elucidated the relationship between facility characteristics and full publication. We observed that academic facilities had an improved chance of publication compared with community hospitals. In Japan, obtaining academic positions related to medicine is highly competitive [30,31]. To obtain and remain in a tenured position in an academic facility, Japanese researchers are required to continuously publish their work in peer-reviewed journals. Our results may reflect this situation.

JSA annual meetings include three presentation styles. Usually, JSA reviewers assign the top $10 \%$ of abstracts to excellent abstracts, the following $30 \%$ of abstracts to poster discussions, and remaining abstracts to poster presentations. Our analysis also demonstrated that abstracts designated as excellent abstracts were more likely to be published than the other two presentation styles. Similarly, the journal impact factor of the final publications was significantly higher for excellent abstracts than other two presentation styles. Therefore, our results suggested that abstract reviewers discern the scientific value of abstracts well, and JSA meeting presentation designation may be used as a "test" to predict the fate of abstracts.

Consistent with prior studies [3, 6, 11], meeting abstracts reporting on basic research were more likely to be published than clinical research. In most cases, experimental studies require careful forethought and planning, funding, a fully equipped laboratory, and protected research time. We assumed that such factors collectively lead to successful publication. Multi-institutional collaboration has increased and gained importance in the domain of scientific research over the last few decades $[32,33]$. This study showed that the number of collaborating facilities was associated with an increased chance of publication. Our results were consistent with a previous report by Larivière et al. [34] that found an increased number of collaborating facilities led to increased research impact.

Our study had several limitations. First, the retrospective bibliometric design meant that we could not attribute causality from the associations between any exposures and publication. Second, as with any observational study, detected associations among publication and presentation style, research type, and first author and affiliation characteristics may be confounded by other factors. Although adjustments were made using a logistic regression model, there might have been other unmeasured confounders. For example, author's age, clinical experience, workload, job title, academic degree, marital status, and number and age of children might have affected the publication rate. Further analyses including such in-depth personal information are required to further clarify factors 
TA B L E 2. Factors associated with subsequent full publication ${ }^{a}$ of research abstracts initially presented at the Japanese Society of Anesthesiologists annual meetings 2015-2017.

\begin{tabular}{|c|c|c|c|c|}
\hline & \multicolumn{2}{|c|}{ Univariable analysis } & \multicolumn{2}{|c|}{ Multivariable analysis ${ }^{b}$} \\
\hline & OR $(95 \%$ CI $)$ & $\boldsymbol{P}$ & $\operatorname{AOR}(95 \% \mathrm{CI})$ & $\boldsymbol{P}$ \\
\hline \multicolumn{5}{|l|}{ Presentation style } \\
\hline Poster presentations & 1 (Reference) & & 1 (Reference) & \\
\hline Poster discussions & $1.85(1.47-2.32)$ & $<0.001$ & $1.70(1.31-2.20)$ & $<0.001$ \\
\hline Excellent abstracts & $5.57(4.18-7.44)$ & $<0.001$ & $2.82(1.98-4.01)$ & $<0.001$ \\
\hline \multicolumn{5}{|l|}{ Presentation topics } \\
\hline Cardiovascular medicine & 1 (Reference) & & 1 (Reference) & \\
\hline Respiratory medicine & $1.07(0.69-1.67)$ & 0.756 & $0.90(0.54-1.51)$ & 0.687 \\
\hline Neuroscience & $3.83(2.36-6.24)$ & $<0.001$ & $1.72(0.95-3.11)$ & 0.073 \\
\hline Pediatric anesthesia & $0.50(0.25-0.98)$ & 0.045 & $0.56(0.27-1.20)$ & 0.137 \\
\hline Obstetric anesthesia & $0.58(0.34-1.00)$ & 0.051 & $0.74(0.40-1.35)$ & 0.327 \\
\hline Pain medicine & $0.83(0.58-1.20)$ & 0.328 & $0.71(0.46-1.08)$ & 0.110 \\
\hline ICU and emergency medicine & $1.60(1.03-2.48)$ & 0.035 & $1.00(0.60-1.68)$ & 0.996 \\
\hline Anesthesia-related topics & $0.85(0.61-1.18)$ & 0.325 & $0.75(0.51-1.10)$ & 0.139 \\
\hline General topics in anesthesia & $0.39(0.24-0.65)$ & $<0.001$ & $0.47(0.27-0.81)$ & 0.007 \\
\hline \multicolumn{5}{|l|}{ Research type } \\
\hline Clinical research & 1 (Reference) & & 1 (Reference) & \\
\hline Basic research & $7.73(6.02-9.92)$ & $<0.001$ & $4.39(3.23-5.96)$ & $<0.001$ \\
\hline \multicolumn{5}{|l|}{ Number of author(s) } \\
\hline One & 1 (Reference) & & 1 (Reference) & \\
\hline Two & $1.58(0.63-3.94)$ & 0.327 & $1.46(0.49-4.29)$ & 0.496 \\
\hline Three & $2.21(0.95-5.11)$ & 0.064 & $1.38(0.50-3.78)$ & 0.536 \\
\hline Four & $3.59(1.58-8.15)$ & 0.002 & $2.57(0.99-6.67)$ & 0.053 \\
\hline Five & $2.80(1.24-6.34)$ & 0.013 & $2.05(0.76-5.48)$ & 0.155 \\
\hline Six & $2.01(0.91-4.44)$ & 0.085 & $1.59(0.60-4.16)$ & 0.350 \\
\hline \multicolumn{5}{|l|}{ Gender of the first author } \\
\hline Male & $1.35(1.09-1.66)$ & 0.007 & $1.41(1.09-1.81)$ & 0.008 \\
\hline Female & 1 (Reference) & & 1 (Reference) & \\
\hline \multicolumn{5}{|l|}{ Number of author affiliation(s) } \\
\hline One & 1 (Reference) & & 1 (Reference) & \\
\hline Two & $2.00(1.60-2.51)$ & $<0.001$ & $1.50(1.15-1.97)$ & 0.003 \\
\hline Three & $2.33(1.68-3.24)$ & $<0.001$ & $1.83(1.23-2.73)$ & 0.003 \\
\hline Four & $2.89(1.65-5.06)$ & $<0.001$ & $2.40(1.27-4.54)$ & 0.007 \\
\hline Five or more & $3.20(1.25-8.22)$ & 0.015 & $2.30(0.74-7.15)$ & 0.150 \\
\hline \multicolumn{5}{|l|}{ First author's affiliation type } \\
\hline Private community hospital & 1 (Reference) & & 1 (Reference) & \\
\hline Public community hospital & $1.23(0.78-1.95)$ & 0.368 & $1.17(0.72-1.90)$ & 0.520 \\
\hline Private university & $5.08(3.49-7.40)$ & $<0.001$ & $3.97(2.60-6.08)$ & $<0.001$ \\
\hline Public university & $5.92(4.11-8.51)$ & $<0.001$ & $3.53(2.35-5.30)$ & $<0.001$ \\
\hline Medical company & $44.74(11.81-169.43)$ & $<0.001$ & $16.70(3.75-74.46)$ & $<0.001$ \\
\hline \multicolumn{5}{|l|}{ First author's affiliation region } \\
\hline Kanto and Koshinetsu & 1 (Reference) & & 1 (Reference) & \\
\hline Hokkaido and Tohoku & $1.05(0.70-1.58)$ & 0.805 & $1.12(0.69-1.80)$ & 0.655 \\
\hline Tokai and Hokuriku & $0.83(0.58-1.19)$ & 0.312 & $0.71(0.46-1.09)$ & 0.115 \\
\hline Kansai & 1.45 (1.11-1.89) & 0.007 & $2.16(1.57-2.99)$ & $<0.001$ \\
\hline Chugoku and Shikoku & $1.13(0.79-1.60)$ & 0.512 & $1.26(0.81-1.97)$ & 0.306 \\
\hline Kyushu and Okinawa & $0.56(0.37-0.85)$ & 0.007 & $0.47(0.29-0.76)$ & 0.002 \\
\hline Abroad & $5.55(3.43-8.98)$ & $<0.001$ & $4.57(2.58-8.09)$ & $<0.001$ \\
\hline
\end{tabular}




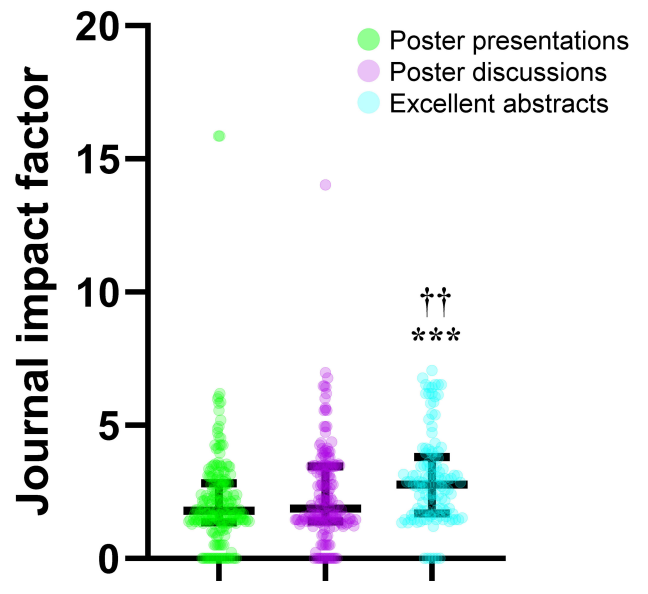

$\begin{array}{llll}\text { Number of published } & 193 & 173 & 121\end{array}$ abstracts

F I G U R E 5. Journal impact factor for published research abstracts presented at the Japanese Society of Anesthesiologists annual meetings 2015-2017 by presentation style. Column scatter plots represent the data distribution (circles), median (horizontal bar), and interquartile range (vertical bar). $P$-values were derived from Kruskal-Wallis tests followed by Dunn's post hoc tests with Bonferroni correction. ***P $<0.001$ vs. poster presentations; $\dagger \uparrow P<0.01$ vs. poster discussions.

associated with successful publication. Finally, because we searched for published articles in a single database (MEDLINE) using a single search engine (PubMed $®)$, some peerreviewed manuscripts published after JSA annual meetings might have been overlooked. The publication rate detected by this study is therefore likely to be lower than the actual publication rate. However, we believe our search strategy was natural and clinically relevant because most researchers initially use MEDLINE as the most reliable source of medical knowledge. We also feel that it is unlikely that a significant difference would have been found even if other databases (e.g., Embase ${ }^{\circledR}$ and Google Scholar ${ }^{\circledR}$ ) were included. For example, Berry et al. [35] found that $94 \%$ of target references were identified in their MEDLINE search for articles included in systematic review of medical imaging.

Despite these limitations, we believe our study provides an accurate depiction of the current anesthesia research activity in Japan. This study included enough abstracts to ensure model stability. Our logistic model was robust with high discrimination ability (c-statistic $>0.8$ ), and successfully detected factors predictive of publication. Further research should seek to clarify barriers to the publication of findings presented at scientific meetings and identify objective ways to assist the publication process.

\section{Conclusions}

This bibliometric analysis found that approximately onefifth of JSA meeting abstracts are subsequently published in MEDLINE-indexed journals within 3 years after the meeting. Factors independently associated with subsequent full publication are presentation style, research type (basic research), male gender, specific region, affiliation characteristics (university and medical company), and increased number of collaborating facilities. We believe this study advances our understanding of current anesthesiology research output from Japan and provides important information to guide the successful publication of research abstracts. We believe that the implications of our results would be beneficial for both our study population and other settings. Our observations should stimulate further investigations that clarify objective ways to assist the full publication process of scientific meeting abstracts.

\section{AUTHOR CONTRIBUTIONS}

YO conceived the study design, collected the data, conducted the database search and the statistical analyses, drafted the initial manuscript, and takes primary responsibility for the whole paper. MS replicated the database searching after YO and checked the data validity and reproducibility. MS also participated in drafting the paper. As a professional librarian, CS provided critical advice and made substantial contributions to the construction of the database search strategy. CS also supervised the database searching and participated in drafting the paper. KS helped in conceptualizing the study design, critically reviewed the manuscript, and participated in reviewing various drafts. SI and JK supervised the study, and participated in discussing, revising, and editing the manuscript. All authors read and approved the final version of the manuscript.

\section{ETHICS APPROVAL AND CONSENT TO PARTICIPATE}

Current Japanese ethical guidelines do not mandate ethical review for studies analyzing publicly available data. This study was therefore exempt from ethical evaluation by the Institutional Review Board at the authors' facilities. Similarly, as this was a bibliometric analysis that did not involve human subjects, the need for informed consent was waived.

\section{ACKNOWLEDGMENT}

We thank Kazushige Toyama, MD, PhD, Tuneyoshi Seki, MD, $\mathrm{PhD}$, Yusuke Miyazaki, MD, and Yoshihisa Fujinami, MD (from the Department of Disaster and Emergency Medicine, Kobe University, Kobe, Japan) for participating in the related discussion. We also thank Audrey Holmes, MA, from Edanz Group (https://en-author-services.edanz.com/ac) for editing a draft of this manuscript. Finally, we thank Nozomi Ono, MD (Department of Psychiatry, Hoshigaoka Hospital, Koriyama, Japan) for assisting in reviewing the manuscript.

\section{FUNDING}

This research received no specific grant from any funding agency in the public, commercial, or not-for-profit sectors. 


\section{CONFLICT OF INTEREST}

The authors declare that there are no conflicts of interest regarding the publication of this article.

\section{DATA AVAILABILITY}

The data used to support the findings of this study are available from the corresponding author upon request.

\section{REFERENCES}

[1] Petticrew M, Gilbody S, Song F. Lost information? The fate of papers presented at the 40th society for Social Medicine Conference. Journal of Epidemiology \& Community Health. 1999; 53: 442-443.

[2] Stolk P, Egberts ACG, Leufkens HGM. Fate of abstracts presented at five International Conferences on Pharmacoepidemiology (ICPE): 19951999. Pharmacoepidemiology and Drug Safety. 2002; 11: 105-111.

[3] Autorino R, Quarto G, Di Lorenzo G, De Sio M, Damiano R. Are abstracts presented at the EAU meeting followed by publication in peer-reviewed journals? A critical analysis. European Urology. 2007; 51: 833-840.

[4] Scherer RW, Meerpohl JJ, Pfeifer N, Schmucker C, Schwarzer G, von Elm E. Full publication of results initially presented in abstracts. Cochrane Database of Systematic Reviews. 2018; 11: MR000005.

[5] Çekmecelioğlu BT, Kozanhan B, Eren G. Publication rate of abstracts orally presented at the Turkish Society of Anaesthesiology and Reanimation National Congresses. Turkish Journal of Anaesthesiology and Reanimation. 2019; 47: 151-157.

[6] Gerlach B, Shah AM, Lee MT, Wong CA, Sullivan JT, Toledo P. Publication rate of abstracts presented at the society for obstetric anesthesia and perinatology annual meetings 2010-2014. Anesthesia \& Analgesia. 2017; 124: 887-889.

[7] Chong SW, Collins NF, Wu CY, Liskaser GM, Peyton PJ. The relationship between study findings and publication outcome in anesthesia research: a retrospective observational study examining publication bias. Canadian Journal of Anaesthesia. 2016; 63: 682-690.

[8] Toledo P, McLean S, Duce L, Wong CA, Schubert A, Ward DS. Evaluation of the foundation for anesthesia education and research medical student anesthesia research fellowship program participants' scholarly activity and career choices. Anesthesiology. 2016; 124: 11681173

[9] Tyagi A, Chugh V, Kumar S, Sethi A. Presentation of research in anesthesia: culmination into publication? Journal of Anaesthesiology Clinical Pharmacology. 2013; 29: 216-220.

[10] Meininger D, Bück M, Bohlmann S, Weber CF, Strouhal U, Ihlow K, et al. Gone with the wind or... Fate of scientific articles presented at large anesthesia congresses-an update. Der Anaesthesist. 2011; 60: 118-124. (In German)

[11] Castillo J, Garcia-Guasch R, Cifuentes I. Fate of abstracts from the Paris 1995 European Society of Anaesthesiologists meeting. European Journal of Anaesthesiology. 2002; 19: 888-893.

[12] Yentis SM, Campbell FA, Lerman J. Publication of abstracts presented at anaesthesia meetings. Canadian Journal of Anaesthesia. 1993; 40: 632 634.

[13] Meranze J, Ellison N, Greenhow DE. Publications resulting from anesthesia meeting abstracts. Anesthesia \& Analgesia. 1982; 61: 445448.

[14] Hirota K. Anesthesia research in Japan: seeds of recovery and the role of the Journal of Anesthesia. Journal of Anesthesia. 2017; 31: 803-805.

[15] Hirota K. Launch of JA Clinical Reports. Anesthesia research crisis in Japan. Journal of Anesthesia. 2015; 29: 161-163.

[16] Hirota K. A worrying decline in anesthesia journal publications from Japan. Journal of Anesthesia. 2013; 27: 323-324.

[17] Hirota K. The present crisis of the publication activity in anesthesia research in Japan and its countermeasure. Masui. 2015; 64: 17-29. (In Japanese)

[18] Li Z, Qiu L, Wu F, Yang L, Sun Y, Yu W. Scientific publications in anesthesiology journals from East Asia: a 10-year survey of the literature.
Journal of Anesthesia. 2011; 25: 257-262

[19] Scherer RW, Langenberg P, von Elm E. Full publication of results initially presented in abstracts. Cochrane Database of Systematic Reviews. 2018; 11: MR000005.

[20] Walby A, Kelly AM, Georgakas C. Abstract to publication ratio for papers presented at scientific meetings: how does emergency medicine compare? Emergency Medicine. 2001; 13: 460-464

[21] Macmillan CD, Moore AK, Cook RJ, Pedley DK. Abstract-to-publication ratio for papers presented at scientific meetings: a quality marker for UK emergency medicine research. Emergency Medicine Journal. 2007; 24: 425-426.

[22] Miguel-Dasit A, Martí-Bonmatí L, Sanfeliu P, Aleixandre R. Scientific papers presented at the European Congress of Radiology 2000: publication rates and characteristics during the period 2000-2004. European Radiology. 2006; 16: 445-450.

[23] DeMola PM, Hill DL, Rogers K, Abboud JA. Publication rate of abstracts presented at the shoulder and elbow session of the American Academy of Orthopaedic Surgery. Clinical Orthopaedics and Related Research. 2009; 467: 1629-1633.

[24] Demailly Z, Brulard G, Selim J, Compère V, Besnier E, Clavier T. Gender differences in professional social media use among anaesthesia researchers. British Journal of Anaesthesia. 2020; 124: e178-e184.

[25] Yasukawa K, Nomura K. The division of labor by sex among Japanese physicians. Igaku Kyouiku. 2012; 43: 315-319. (In Japanese)

[26] Ueda T, Setoyama K, Oka S, Yamamoto Y. The Present situation of female anesthesiologists-analysis of questionnaire. Journal of Japan Society for Clinical Anesthesia. 2004; 24: 573-578.

[27] Carr PL, Ash AS, Friedman RH, Scaramucci A, Barnett A, Szalacha L, et al. Relation of family responsibilities and gender to the productivity and career satisfaction of medical faculty. Annals of Internal Medicine. 1998; 129: 532.

[28] Yasukawa K, Nomura K. The perception and experience of gender-based discrimination related to professional advancement among Japanese physicians. Tohoku Journal of Experimental Medicine. 2014; 232: 35 42.

[29] Reed DA, Enders F, Lindor R, McClees M, Lindor KD. Gender differences in academic productivity and leadership appointments of physicians throughout academic careers. Academic Medicine. 2011; 86: 43-47.

[30] Ono Y, Goto A, Maejima Y, Maruyama I, Suzuki T, Shikama Y, et al. Work-life conflict, gender-based discrimination, and their associations among professionals in a medical university and affiliated hospitals in Japan: a cross-sectional study. Fukushima Journal of Medical Science. 2020; 66: 25-36

[31] Chatani Y, Nomura K, Horie S, Takemoto K, Takeuchi M, Sasamori $\mathrm{Y}$, et al. Effects of gaps in priorities between ideal and real lives on psychological burnout among academic faculty members at a medical university in Japan: a cross-sectional study. Environmental Health and Preventive Medicine. 2017; 22: 32.

[32] Abramo G, D'Angelo CA. The relationship between the number of authors of a publication, its citations and the impact factor of the publishing journal: evidence from Italy. Journal of Informetrics. 2015; 9: 746-761.

[33] Wuchty S, Jones BF, Uzzi B. The increasing dominance of teams in production of knowledge. Science. 2007; 316: 1036-1039.

[34] Larivière V, Gingras Y, Sugimoto CR, Tsou A. Team size matters: collaboration and scientific impact since 1900. Journal of the Association for Information Science and Technology. 2015; 66: 1323-1332.

[35] Berry E, Kelly S, Hutton J, Harris KM, Smith MA. Identifying studies for systematic reviews. An example from medical imaging. International Journal of Technology Assessment in Health Care. 2000; 16: 668-672.

How to cite this article: Yuko Ono, Masafumi Saito, Chiaki Shinohara, Kazuaki Shinohara, Shigeaki Inoue, Joji Kotani. Factors associated with successful publication of research abstracts presented at the Japanese Society of Anesthesiologists annual meetings 2015-2017: a bibliometric analysis. Signa Vitae. 2021;17(3):85-94. doi:10.22514/sv.2021.036. 